Volume 8, No.6, November -December 2019

International Journal of Science and Applied Information Technology

Available Online at http://www.warse.org/ijsait/static/pdf/file/ijsait22862019.pdf

https://doi.org/10.30534/ijsait/2019/228620198

\title{
Enhanced Handover Management in 4G Mobile Network
}

\author{
Mohammad Al Shinwan, Laith Abualigah, Ahmad M. Khasawneh, Hamzeh Alabool, Deemah Alarabiat \\ Faculty of Computer Sciences and Informatics \\ Amman Arab University \\ Amman, Jordan \\ mohmdsh@aau.edu.jo
}

\begin{abstract}
The current Evolved Packet Core (EPC) 4th generation (4G) mobile network structure emphasizes complicated control plane protocols and requires expensive equipment. In this approach, we propose the creation of a tunnel that maintains data delivery to mobile devices until the new Base Station (BS) element updates the route with the gateway, which prevents data packet loss during handover between BS elements located near to one another. To maintain the handover without losing the data we propose an approach scheme based on IP encapsulated within IP (IP-in-IP) for data delivery. We describe the results of numerical analyses showing that the proposed architecture provides superior performance compared with the current $4 \mathrm{G}$ architecture in terms of handover delay.
\end{abstract}

Key words: 4G network, Handover, LTE, Mobility Management

\section{INTRODUCTION}

Recently, the marked increase in the use of mobile phones and other mobile devices has led to a tremendous increase in mobile communication data traffic. This growth in mobile data traffic places increasing demands on wireless communication systems and represents a major challenge for cellular providers in terms of improving their core networks to support future network demands and keeping up with growing customer requirement [1].

Many methods have been introduced to address the extension in data traffic on mobile communication networks, including device-to-device (D2D) communication and wireless radio resource management. However, these efforts have concentrated essentially on expanding the capacity of wireless radio links. The future mobile network consists of two main parts: a radio link and a non-radio mobile core network. The efficient design of both the mobile core and the radio link is needed to meet the demands of the future mobile network [2].

The current 4th generation (4G) core network termed the Evolved Packet Core (EPC) is based on the General Packet Radio Service tunneling protocol (GTP) [4]. With EPC, eNodeB (eNB) or Base station (Bs) elements establish GTP tunnels with serving gateways (S-GWs) and Packet Data Network (PDN) gateways (P-GWs) to create centralized mobility anchors for data packet forwarding. However, the $4 \mathrm{G}$ network has a number of limitations.
- There are load balance and latency issues. Growth in data traffic requires a reduction in the transmission and connection delays. Simplifying the handover process between Base Station (BS) and decreasing the number of identifications can make handover simpler and more efficient, and hence more cost-effective.

- The GTP tunneling protocol overhead. GTP protocol adds three headers to the data payload totaling 36 bytes (GTP, UDP, and IP).

Various systems have been proposed to overcome these problems. The Distributed Mobility Management (DMM) approach [3] provides mobility solutions with localized mobility anchors distributed within the network. A mobility data offloading approach using femtocells has been introduced to enhance the $4 \mathrm{G}$ network [4]. In this scheme, data traffic is forward to the mobile device without using the mobile core network, which decreases the volume of internet traffic and unwanted data flow into the mobile core network.

However, most existing architectures suffer from problems associated with mobility anchoring and GTP overhead. In addition, from the perspective of capital expenditure, most of the proposed mobile network architectures are not costeffective. Other optimization techniques can be used [8-14].

In this paper we propose a handover model between BSs located near to one another, we propose the use of an IP encapsulated within IP to maintain data flow to mobile devices until the new BSs updates the route with the gateway. In this way, we prevent data packet loss during handover.

The rest of this paper is organized as follows. Section II describes the existing handover management in the $4 \mathrm{G}$ network. In Section III, we demonstrate our proposed approach. We analyze the performances of our approach and the $4 \mathrm{~g}$ network and compare them in Section IV. Numerical results are provided in Section $\mathrm{V}$ and the conclusion follows in Section VI.

\section{HANDOVER MANAGEMENT IN 4G NETWORK}

There are two types of handover process in a $4 \mathrm{G}$ network: $\mathrm{X} 2$ handover with S-GW relocation, and S1 handover with SGW relocation.

\section{A. $X 2$ handover with $S$ - $G W$ relocation}

When the MN moves to another BS region, the source BS sends a Handover Request to the target BS, which response 
with a Handover Acknowledgment. The target BS then sends a Path Switch Request to the MME, which sends a Create Session Request to the S-GW. The S-GW exchanges modify bearer messages with the P-GW via a Modify Bearer Request and a Modify Bearer Response, and then sends a Create Session Response to the MME, which sends a Path Switch Response to the target BS. Finally, the MME sends a Release Resources message to the source BS.

\section{B. S1 handover with $S-G W$ relocation}

When the MN moves to a new domain, the source MN sends a Handover required message to the MME. The MME then sends a Handover Request to target $\mathrm{MN}$, which response with a Handover Acknowledgment. The MME then sends a Handover Command to the source $\mathrm{MN}$, which sends a Handover Notify message to the MME. The MME sends a Modify Bearer Request to the target S-GW, which exchanges modify bearer messages with the P-GW via a Modify Bearer Request and a Modify Bearer Response. The target S-GW sends a Modify Bearer Response to the MME, which then sends a Release Resources message to the source BS.

\section{PROPOSED NETWORK MODEL}

In this paper, we will discuss and modify the $\mathrm{X} 2$ handover with S-GW relocation and proposed a new handover model based on IP encapsulation. This handover procedure occurs between a source BS and a target BS when the MN moves but remains within the same domain. With this operation, there is the risk of data packet loss. When the UE moves to the target $\mathrm{BS}$, the source BS still receives data packets from the P-GW. To bridge this gap, these two BSs communicate with each other directly via IP-in-IP protocol [5]. This supports handover and prevents data packet loss during the handover process.

Fig. 1 demonstrate how the source BS encapsulate the inner IP address with outer IP of target BS to exchange data. During this process, the MN continues to receive data packets from the source BS until handover to the target BS has been completed. Fig. 2 shows the protocol stack for BSs connected via an IP-in-IP. An inner IP is generated for each UE, and each BS has an outer IP.

Fig. 3 shows the handover procedure, during which the MN moves from the source BS to the target BS as a follow:

1) . The source BS sends a Handover Request to the target BS, which response with a Handover Acknowledgment, thereby the IP packet encapsulate within outer IP.

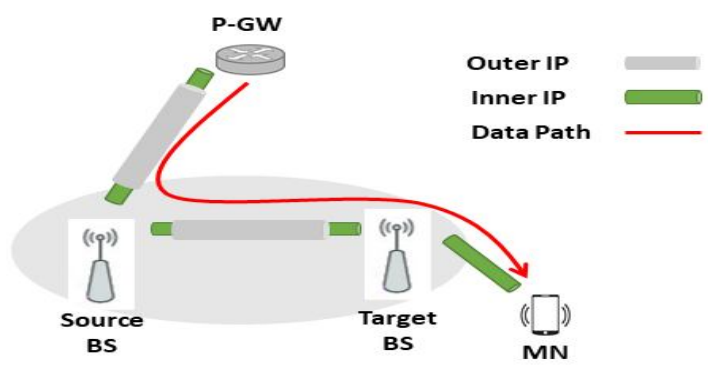

Figure 1: Source BS encapsulate the inner IP address with outer IP of target BS

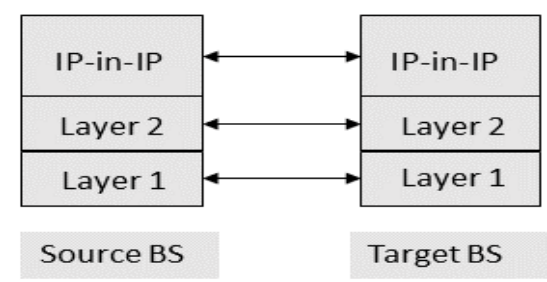

Figure. 2: The IP-in-IP protocol packet stack for BSs.

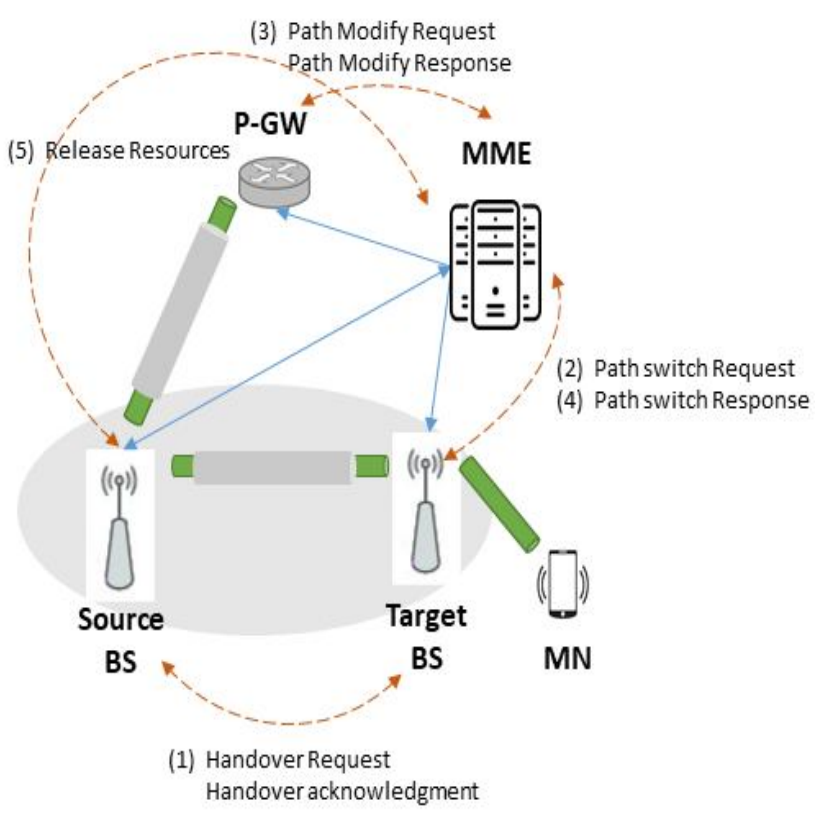

Figure 32: Handover procedure

2) The target BS sends a Path Switch Request to the MME.

3) The MME exchanges Path Switch Response and Request message with P-GW, to inform the MME that the MN has moved to a new BS, and the P-GW sends a Path Modify Response to the MME. Finally, the target BS sends a Release Resources message to the source BS.

\section{NUMERICAL ANALYSIS}

We calculated the total transmission delay of current $4 \mathrm{G}$ network and the proposed model based on the hop count between the BSs and between the BS and MME unite.

We determined the transmission delay of a message with size $S$ that was sent between two nodes over a wireless link and a wired link [6].

The transmission delay of a message with size $S$ sent via a wireless link from $\mathrm{x}$ to $\mathrm{y}$ as $\mathrm{T}(\mathrm{x}-\mathrm{y})(\mathrm{S})$, can be represented as follows:

$T_{x-y}(S)=[(1-q) /(1+q)] \times\left[S / B_{w l}+L_{w l}\right]$

We express the transmission delay of the message with size $\mathrm{S}$ sent via a wired link from $\mathrm{x}$ node to y node as $\mathrm{T}_{x-y}\left(\mathrm{~S}, \mathrm{H}_{x-y}\right)$, 
where Hx-y denotes the number of wired hops between $\mathrm{x}$ and y. $\mathrm{T}_{x-y}\left(\mathrm{H}_{x-y}\right)$ can be expressed as follows:

$$
T_{x-y}\left(S, H_{x-y}\right)=H_{x-y} \times\left[\left(S / B_{w}\right)+L_{w}+T_{q}\right]
$$

In the performance analysis, we used the notation and default parameter values listed in Table 1.

Based on the handover procedure that discussed in section II, the total $\mathrm{X} 2$ handover delay of the $4 \mathrm{G}$ network presented as follows:

$$
2 T_{B S \sim B S}+3 T_{B S \sim M M E}+2 T_{M M E \sim S-G W}+2 T_{S-G W \sim P-G W}
$$

We obtain the inter-gateway handover delay of the proposed network as follows:

$$
3 T_{B S \sim B S}+2 T_{B S \sim M M E}+2 T_{M M E \sim S-G W}
$$

\section{NUMERICAL RESULTS OF HANDOVER DELAY}

Based on the mathematical functions given above, we compare the performance of the current $4 \mathrm{G}$ network handover with the proposed architecture. Table 1 lists the default values of the delay parameters [7].

As shown in fig. (4) a comparison of proposed handover and $4 \mathrm{G}$ network handover. This comparison is based on the hop count between BSs in the same domain. The handover between BSs had a greater impact on the handover delay for the $4 \mathrm{G}$ network. This is because the MME changes the bearer via a centralized anchor (S-GW/P-GW), whereas the proposed approach is largely insensitive to BS handover because the MME performs path modification with the P-GW.

Fig. (5) shows a comparison of S1 handover with S-GW relocation. This comparison was based on the hop count between the BS and MME. The result show a significant impact on the handover delay for the $4 \mathrm{G}$ network; this is because the MME implements modify bearer with the P-GW via the S-GW. By contrast, with the proposed approach network, the MME performs path switching directly with MME.

Tablke 1: Default parameter values

\begin{tabular}{|l|l|c|}
\hline Parameter & \multicolumn{1}{|c|}{ Description } & Value \\
\hline Lwl & Delay of a wirless link & $10 \mathrm{~ms}$ \\
\hline Lw & Delay of a wired link & $2 \mathrm{~ms}$ \\
\hline $\mathrm{q}$ & Wireless Link failure & 0.2 \\
\hline $\mathrm{Tq}$ & $\begin{array}{l}\text { Average queuing delay at each } \\
\text { node }\end{array}$ & $5 \mathrm{~ms}$ \\
\hline $\mathrm{Sc}$ & Size of control packets & $50 \mathrm{bytes}$ \\
\hline $\mathrm{Sd}$ & Size of data packets & $200 \mathrm{bytes}$ \\
\hline $\mathrm{Bwl}$ & Bandwidth of wireless links & $11 \mathrm{Mbps}$ \\
\hline Bw & Bandwidth of wired links & $100 \mathrm{Mbps}$ \\
\hline
\end{tabular}

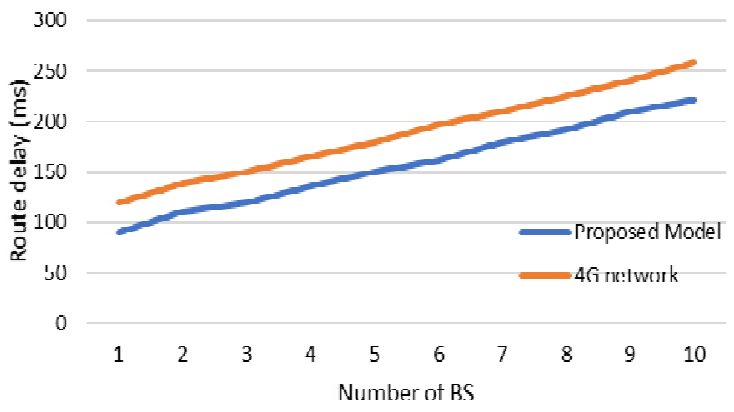

Figure 3: The effect of hop count on the handover delay

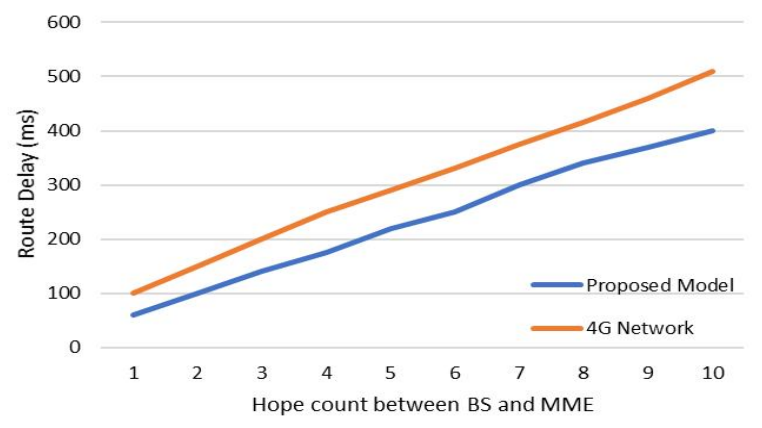

Figure 4: The effect of hop count between BS and MME on the handover delay.

\section{CONCLUSION}

The current $4 \mathrm{G}$ mobile handover process is inflexible. We have described a handover model, which is based on IP-in-IP protocols for data packet delivery. With this architecture, data packets are delivered between BSs near to each other via outer IP. The proposed model achieves better performance than $4 \mathrm{G}$ by eliminates the GTP tunneling protocol and using IP-in-IP.

We compared our approach architecture with the existing $4 \mathrm{G}$ network architecture using numerical analyses. The results show that the proposed model results in better performance than the $4 \mathrm{G}$ network in terms of the handover delay.

\section{REFERENCES}

[1] Al Shinwan, Mohammad, and Kim Chul-Soo. "A future mobile packet core network based on ip-in-ip protocol." International Journal of Computer Networks \& Communications (IJCNC) Vol 10 (2018).K. Elissa, “Title of paper if known," unpublished.

[2] Al Shinwan, Mohammad, Trong-Dinh Huy, and Kim Chul-Soo. "A Flat Mobile Core Network for Evolved Packet Core Based SAE Mobile Networks." Journal of Computer and Communications 5, no. 05 (2017): 62.

[3] Bertin, Philippe, Servane Bonjour, and Jean-Marie Bonnin. "An evaluation of dynamic mobility anchoring." 
In 2009 IEEE 70th Vehicular Technology Conference Fall, pp. 1-5. IEEE, 2009.

[4] Rebecchi, Filippo, Marcelo Dias De Amorim, Vania Conan, Andrea Passarella, Raffaele Bruno, and Marco Conti. "Data offloading techniques in cellular networks: A survey." IEEE Communications Surveys \& Tutorials 17, no. 2 (2015): 580-603.

[5] Perkins, Charles. IP encapsulation within IP. No. RFC 2003. 1996.

[6] Makaya, Christian, and Samuel Pierre. "An analytical framework for performance evaluation of IPv6-based mobility management protocols." IEEE Transactions on wireless communications 7, no. 3 (2008): 972-983.

[7] Shinwan, M. A., and K. Chul-Soo. "Enhanced Mobile Packet Core Network Scheme for Next-Generation Mobile Communication Systems." International Journal of Electronics Communication and Computer Engineering (IJECCE) 8 (2017): 56-61.

[8] Abualigah, L. M. Q. (2019). Feature selection and enhanced krill herd algorithm for text document clustering. Berlin: Springer. https://doi.org/10.1007/978-3-030-10674-4

[9] Abualigah, L. M., Khader, A. T., \& Hanandeh, E. S. (2019). Modified Krill Herd Algorithm for Global Numerical Optimization Problems. In Advances in
Nature-Inspired Computing and Applications (pp. 205221). Springer, Cham.

[10] Abualigah, L. M., \& Khader, A. T. (2017). Unsupervised text feature selection technique based on hybrid particle swarm optimization algorithm with genetic operators for the text clustering. The Journal of Supercomputing, 73(11), 4773-4795.

[11] Abualigah, L. M., Khader, A. T., Hanandeh, E. S., \& Gandomi, A. H. (2017). A novel hybridization strategy for krill herd algorithm applied to clustering techniques. Applied Soft Computing, 60, 423-435.

[12] Abualigah, L. M., Khader, A. T., \& Hanandeh, E. S. (2018). Hybrid clustering analysis using improved krill herd algorithm. Applied Intelligence, 48(11), 4047-4071.

[13] Abualigah, L. M., Khader, A. T., \& Hanandeh, E. S. (2018). A new feature selection method to improve the document clustering using particle swarm optimization algorithm. Journal of Computational Science, 25, 456466.

[14] Abualigah, L. M., Khader, A. T., \& Hanandeh, E. S. (2018). A combination of objective functions and hybrid Krill herd algorithm for text document clustering analysis. Engineering Applications of Artificial Intelligence, 73, $111-125$

https://doi.org/10.1016/j.engappai.2018.05.003 